\title{
Association Between Prevalence of Anaemia and Malaria in Under-Five of Uganda: A Comparison of Ordered and Binary Logistic Multilevel Models
}

Charles Natuhamya ( $\sim$ natuhamyac@gmail.com )

Tryphena Nareeba

Fredrick Makumbi

John M. Ssenkusu

David Guwatudde

Nazarius Mbona Tumwesigye

Mary Nakafeero

Research Article

Keywords:

Posted Date: March 7th, 2022

DOI: https://doi.org/10.21203/rs.3.rs-1270863/v1

License: (9) This work is licensed under a Creative Commons Attribution 4.0 International License.

Read Full License 


\section{Abstract}

Background: several analyses of anemia prevalence in multilevel settings have dichotomized anemia as the response variable, ignoring its distinct ordered levels, leading to loss of information that may produce biased estimates. This study aimed at examining estimates from two multilevel models and the association between anemia and malaria in under-five of Uganda.

Methods: a sample of 7,632 children, aged 0-59 months from the 2018-19 malaria indicator survey was analyzed using multilevel mixed effects logistic regression models. In one model, anemia was modeled as an ordinal categorical variable (no anemia, mild, moderate and severe) while in another model as a binary variable (no anemia and anemia). The main explanatory variable was the result of malaria test. Five models were fitted using each method (Model-1: the null model, Model-2: with malaria test result as the only covariate, Model-3: with individual level factors, Model-4: with household level factors, Model-5: with cluster level factors). The final model from each method was selected using Akaike's information criterion (AIC). The model with more reliable estimates was selected basing on standard error (SE) values. Random effects variables were region, cluster and household.

Results: anemia prevalence was (overall $=51.62 \%$; severe $=1.36 \%$; moderate $=25.00 \%$; mild $=25.26 \%$ ). The likelihood of a child who tested positive for malaria being anemic was about 5 folds in both models $(\mathrm{OR}=5.005 ; 95 \% \mathrm{Cl}, 4.369-5.733 ; \mathrm{p}<0.001)$ and $(\mathrm{OR}=4.697 ; 95 \% \mathrm{Cl}, 3.983-5.538 ; \mathrm{p}<0.001)$ for the ordered and binary logistic model respectively. Generally; sex, age, and wealth index were associated with a lower likelihood of being anemic. Although AIC values were lower in the binary model compared to the ordered model, the former was considered as a better model as it yielded lower standard errors for all estimates hence more reliable values.

Conclusion: analysts should use modeling techniques that account for the order aspect in categorical outcome variables and avoid dichotomization. Choice of the best model should be based on standard errors besides AIC. Large standard errors are associated with large confidence intervals that may lead to type II error. Malaria increases risk of anemia.

\section{Introduction}

Anaemia is a condition in which the number of red blood cells (and consequently their oxygen-carrying capacity) is insufficient to meet the body's physiologic needs (WHO, Nutritional anaemias, 1968). Generally, it is a reduced level of haemoglobin in the blood. Malaria is one of the leading causes of anaemia that is associated with impared motor and cognitive development in children among other complications (NMCD, UBOS, \& ICF, 2020). About 8 percent of all (24 million) children who were infected with malaria in 2018 in sub-Saharan Africa had severe anaemia. Anaemia prevalence in children under 5 years who tested positive according to a rapid diagnostic test (RDT) was double that of children with a negative RDT in 21 moderate to high malaria burden African countries (WHO, 2019). Anaemia is usually 
measured by categorizing haemoglobin into an ordered variable with four levels. But some analysts often go on to recategorise it into a binary variable to simplify analysis.

Despite of the negative consequences, many researchers continue to dichotomize ordered categorical outcome variables to measure individual differences. In the process of dichotomizing a variable, one may seriously underestimate the extent of variation in an outcome between groups, such as the risk of some event, and considerable variability may be subsumed within each group. Individuals close to but on opposite sides of the cut point are characterized as being very different rather than very similar as the case of ordered categorical outcomes. (Altman \& Royston, 2006). This results into loss of information about individual differences; loss of power, false statistical significance, overestimation of effect size and loss of measurement reliability. (MacCallum, Zhang, Preacher, \& Rucker, 2002). Remarkably, the degree of dissimilarity among the adjacent levels of the scale in an ordinal variable might not necessarily be always the same. (Fernandez, Liu, \& Costilla, 2019)

This study used data collected in the Uganda Malaria Indicator Survey (UMIS) to determine the association between anemia and malaria among under-five children in Uganda. UMIS sample is the result of a multi-stage stratified design, and consequently it was necessary to use more complex formulae. (NMCD, UBOS, \& ICF, 2020). In these data, anemia (the outcome variable of interest) is tested by measuring hemoglobin $(\mathrm{Hb})$ level on a continuous scale in grams per deciliter $(\mathrm{g} / \mathrm{dl})$. Several researchers have dichotomized hemoglobin into (0-not anemic, 1-anaemic) neglecting the recommended ordered grouping (not anemic, mild, moderate and severe) for example; (Ngnie-Teta, Receveur, \& Kuate-Defo, 2007) and (Prieto-Patron, Horst, Hutton, \& Detzel, 2018). Notably, there are advantages when a model developed for modeling ordinal data is used such as avoiding "floor" and "ceiling" effects and avoiding assigning scores, as it happens in continuous models, which can produce results sensitive to the score assigned. (Fernandez, Liu, \& Costilla, 2019).

The main objective of the study was to determine the effect of dichotomizing ordered categorical outcome variables with more than two levels on estimation of model parameters in a multilevel setting.

\section{Methods}

\section{Data Source and Study Population}

This study used secondary data from the most recent Uganda Malaria Indicator Survey (MIS) of 2018/19. The 2018/19 Uganda MIS used a two-stage cluster and stratified sampling technique qualifying it as a survey based on a complex design. The study population consists of children less than 5 years of age who were tested for anaemia and malaria infection. Blood samples for biomarker testing were collected by finger- or heel-prick from children age 0-59 months. Each field team included two health technicians who carried out the anaemia and malaria testing and prepared the blood smears (NMCD, UBOS, \& ICF, 2020) 


\section{Ordered and Binary Logistic Multilevel Regression Models}

This study used two analysis methods; ordered and binary logistic multilevel regression models while determining the association between anemia and malaria. Five models were fitted using each method (Model-1: the null model, Model-2: with malaria test result as the only covariate, Model-3: with individual level factors, Model-4: with household level factors, Model-5: with cluster level factors). Model comparison was done using Akaike's information criterion (AIC) to select the final model from each method. However, the model with the most reliable estimates was based on standard error (SE) values. Model selection was based on lower values both for AIC and SE. The level of significance was set at 0.05 and STATA 15.0 was used for all analyses. Besides modeling probabilities in the binary logistic model (Equation 1), we modeled two cumulative probabilities in ordered logistic, one less-than or equal to $\mathrm{j}$ and the other greater-than $\mathrm{j}$ (equation 2 ). $\mathrm{X}$ and $\mathrm{Y}$ represent covariates and the outcome variable (anaemia level) respectively.

$$
\begin{aligned}
& \operatorname{logit}(\mathrm{p})=\log \frac{\mathrm{P}(\mathrm{Y}=1 \backslash \mathrm{X})}{1-\mathrm{P}(\mathrm{Y}=1 \backslash \mathrm{X})} \\
& \operatorname{logit}[(\mathrm{Y} \leq \mathrm{j} \backslash \mathrm{X})]=\log \frac{\mathrm{P}(\mathrm{Y} \leq \mathrm{j} \backslash \mathrm{X})}{1-\mathrm{P}(\mathrm{Y} \leq \mathrm{j} \backslash \mathrm{X})}
\end{aligned}
$$

\section{Results}

\section{Descriptive Characteristics}

Of the 7,632 children considered in this study, $21.1 \%$ tested positive for malaria by RDT. The child's sex was evenly distributed $(50.7 \%$ and $49.3 \%)$ for male and female respectively. Most mothers had attained primary education $(57.4 \%)$ and most households were headed by males $(71.8 \%)$. Most households were in rural areas (72.3\%), in a low wealth index (57.5\%) and had no piped water sources (93.0\%).

\section{Table 1: Distribution of Under-Five Children by Selected Background Characteristics}




\begin{tabular}{|llll|}
\hline Characteristic & Category & Count & Percent \\
\hline Malaria RDT & Negative & 6,021 & 78.89 \\
\hline Sex of child & Positive & 1,611 & 21.11 \\
\hline Mother's education level & Male & 3,870 & 50.71 \\
\hline & Female & 3,762 & 49.29 \\
\hline Primary & 1,356 & 21.33 \\
\hline Wealth index & At least secondary & 1,355 & 21.31 \\
\hline & Low & 4,385 & 57.46 \\
\hline Place of residence & Middle & 1,229 & 16.10 \\
\hline \multirow{2}{*}{ Source of drinking water } & High & 2,018 & 26.44 \\
\hline & Urban & 1,453 & 19.04 \\
\hline Sex of household head & Rural & 5,518 & 72.30 \\
\hline & Refugee & 661 & 8.66 \\
\hline & Male & 5,480 & 71.80 \\
\hline & Female & 2,152 & 28.20 \\
\hline & 7,097 & 92.99 \\
\hline
\end{tabular}

\section{Multivariable Models}

The World Health Organization (WHO) defines mild anemia as a Hb concentration of between $10 \mathrm{~g} / \mathrm{dL}$ and $10.9 \mathrm{~g} / \mathrm{dL}$, moderate anemia as between $7 \mathrm{~g} / \mathrm{dL}$ and $9.9 \mathrm{~g} / \mathrm{dL}$, and severe anemia as below $7 \mathrm{~g} / \mathrm{dL}$ (WHO, 2019). The outcome variable under ordered logistic multilevel model was classified following this ordering as opposed to that of the binary model. Of the 7,632 children considered in this study, $48.4 \%$ were not anemic, $51.6 \%$ had any anemia; $25.3 \%$ had mild, $25.0 \%$ had moderate and $1.4 \%$ had severe anemia. The likelihood of a child who tested positive for malaria being anemic was about 5 folds in both models (Adjusted Odds Ratio, AOR=5.005; 95\%Cl, $4.369-5.733 ; \mathrm{p}<0.001)$ and (AOR=4.697; 95\% $\mathrm{Cl}, 3.983$ - 5.538; $p<0.001)$ for the ordered and binary logistic models respectively. Generally; sex, age, and wealth index (WI) were associated with a lower likelihood of being anemic. 
Table 2: Estimated odds ratios of ordered and binary logistic multilevel regression models on anaemia.

\begin{tabular}{|c|c|c|c|c|c|c|c|c|c|c|}
\hline \multirow[t]{2}{*}{ Variable } & \multicolumn{5}{|c|}{ Ordered Logistic Multilevel Model } & \multicolumn{5}{|c|}{ Binary Logistic Multilevel Model } \\
\hline & \multirow[t]{2}{*}{ AOR } & \multirow[t]{2}{*}{ SE } & \multirow[t]{2}{*}{$\mathbf{P}$} & \multicolumn{2}{|c|}{$(95 \% \mathrm{Cl})$} & \multirow[t]{2}{*}{ AOR } & \multirow[t]{2}{*}{ SE } & \multirow{2}{*}{$\mathbf{P}$} & \multicolumn{2}{|c|}{$(95 \% \mathrm{Cl})$} \\
\hline $\begin{array}{l}\text { Malaria } \\
\text { RDT }\end{array}$ & & & & & & & & & & \\
\hline Positive & 5.005 & 0.347 & $<0.001$ & 4.369 & 5.733 & 4.697 & 0.395 & $<0.001$ & 3.983 & 5.538 \\
\hline \multicolumn{11}{|l|}{$\begin{array}{l}\text { Sex of } \\
\text { child }\end{array}$} \\
\hline Female & 0.776 & 0.039 & $<0.001$ & 0.704 & 0.856 & 0.797 & 0.045 & $<0.001$ & 0.713 & 0.891 \\
\hline $\begin{array}{l}\text { Age of } \\
\text { child }\end{array}$ & 0.602 & 0.012 & $<0.001$ & 0.579 & 0.626 & 0.596 & 0.014 & $<0.001$ & 0.569 & 0.624 \\
\hline \multicolumn{11}{|l|}{$\begin{array}{l}\text { Wealth } \\
\text { index }\end{array}$} \\
\hline Poorer & 0.792 & 0.056 & $<0.001$ & 0.689 & 0.911 & 0.807 & 0.066 & 0.009 & 0.687 & 0.947 \\
\hline Middle & 0.812 & 0.065 & 0.010 & 0.693 & 0.951 & 0.810 & 0.074 & 0.022 & 0.676 & 0.969 \\
\hline Richer & 0.661 & 0.057 & $<0.001$ & 0.558 & 0.783 & 0.651 & 0.063 & $<0.001$ & 0.539 & 0.786 \\
\hline Richest & 0.843 & 0.073 & 0.048 & 0.711 & 0.998 & 0.836 & 0.081 & 0.065 & 0.691 & 1.011 \\
\hline $\begin{array}{l}\text { Cluster } \\
\text { altitude }\end{array}$ & 1.000 & 0.000 & 0.365 & 1.000 & 1.000 & 1.000 & 0.000 & 0.032 & 1.000 & 1.000 \\
\hline AIC & 15278 & & & & & & & 9270 & & \\
\hline
\end{tabular}

The standard error is an important indicator of how precise an estimate of the population parameter the sample statistic is. That is, it represents the degree of precision with which the sample statistic represents the population parameter. The smaller the standard error, the closer the sample statistic is to the population parameter. (McHugh, 2008). It is evident that although AIC values were lower in the binary model compared to the ordered model as shown in Figure 1, the ordered model yielded lower standard errors for all estimates as opposed to the binary model (Figure 2). Hence the ordered model produced more reliable estimates compared to the binary one and was considered as a better model.

Notably, large standard errors are associated with wide confidence intervals as indicated in Table 1. Wide confidence intervals affect precision leading to unnecessarily failing to reject the null hypothesis that is, creating type II error. 


\section{Discussion}

A mixed-effects regression model that allows an ordinal response was compared to one of a binary outcome, both based on a three-level data structure. The models allowed random effects to be included at the second and third level of clustering.

This study presented that a higher proportion of children who tested positive for anaemia had a higher prevalence of malaria (29.95\%) compared to those who were not anaemic $(11.67 \%)$ on average. Moreover, children who tested positive for malaria had about 5 folds odds of testing being anaemic. Previous studies like (Ngnie-Teta, Receveur, \& Kuate-Defo, 2007) have also shown results similar to our study concerning the relationship between anaemia and malaria.

Some studies suggest that malaria control methods can significantly reduce anaemia cases like (NgnieTeta, Receveur, \& Kuate-Defo, 2007), (Prieto-Patron, Horst, Hutton, \& Detzel, 2018) and (UNICEF, Key practice: Malaria prevention, 2021). As an example, (UNICEF, Key practice: Malaria prevention, 2021) found out that sleeping under insecticide treated nets every night helps to control anaemia in children and adults, as well as reducing household expense on treatment and funeral expenses (UNICEF, Key practice: Malaria prevention, 2021).

Basing on the results from the compared modeling methods, it should be noted that modeling anaemia as an ordered outcome produces better results as compared to dichotomizing it. Hence researchers should use the recommended categorisisation by WHO for haemoglobin levels to diagnose anaemia at sea level $(\mathrm{g} / \mathrm{l})$. That is; 110 or higher for non-anaemia, 100-109 for mild, 70-99 for moderate and lower than 70 for severe anaemia (WHO, Nutritional anaemias, 1968) to avoid potential loss of information (MacCallum, Zhang, Preacher, \& Rucker, 2002) which leads to commission of type II error like the case of results from this study.

\section{Study strengths and limitations}

The multilevel design of the MIS that allows contextual analysis was the strength of this study. The limitations were; parasitaemia was diagnosed using RDT which is not the gold standard. Also, given that the data resulted from a cross-sectional design, causal relationship between explanatory variables and anaemia could not be guaranteed. This was a drawback concerning the prediction capability of the final model. In addition, some variables deemed important at various levels of the nested data were not among collected data for example, feeding variables and sampling weights that correspond to the cluster level of the two-stage sampling design were not provided in the datasets. Also, variables that could be used in computation of these weights were missing. This can result into estimates from which inference at a national level can not be drawn. Another limitation was potential recall bias because household and children's characteristics were purely based on self-report by survey respondents. 


\section{Conclusion}

Neglecting the order aspect in an outcome variable while modeling categorical variables leads to loss of information. Dichotomization treats all units on one side of the cut point similar to each other but completely different from their counterparts. Wider confidence intervals are partly as a result of large standard errors that may lead to unnecessarily failing to reject the null hypothesis hence type II error (wrong inference) since the formula for the $95 \%$ confidence interval for odds ratio as an example is; $\exp (\log (O R)) \pm[1.96 * S E(\log (O R))]$.

Therefore analysts ought to use modeling techniques that account for the order aspect in an ordered categorical outcome variable instead of dichotomizing it to ease analysis and interpretation of results. But choosing to use ordered categorical outcomes might come at a cost because reducing the standard error of requires increasing sample size to increase records for each category in the ordered outcome as

$S E\left((\log (O R))=\sqrt{\frac{1}{a}+\frac{1}{b}+\frac{1}{c}+\frac{1}{d}}\right.$

for a case control where $a, b$ are counts for cases in exposed and unexposed and $c, d$ are counts for controls in exposed and unexposed arms respectively. Hence, the choice of the best model should not always be based on AIC values alone, standard error values should be considered as well.

\section{References}

Altman, D. G., \& Royston, P. (2006). The cost of dichotomising continuous variables. British medical journal, 332:1080.

Fernandez, D., Liu, I., \& Costilla, R. (2019). A method for ordinal outcomes: The ordered stereotype model. International Journal of Methods in Psychiatric Research.

MacCallum, R. C., Zhang, S., Preacher, K. J., \& Rucker, D. D. (2002). On the Practice of Dichotomization of Quantitative Variables. Psychological Method, 7(1), 19-40.

McHugh, M. L. (2008). Standard error: meaning and interpretation. Biochemia Medica, 18(1):7-13.

Ngnie-Teta, I., Receveur, O., \& Kuate-Defo, B. (2007). Risk factors for moderate to severe anemia among children in Benin and Mali: Insights from a multilevel analysis. Food and Nutrition Bulletin, 28(1).

NMCD, UBOS, \& ICF. (2020). Uganda Malaria Indicator Survey 2018-19. Kampala, Uganda, and Rockville, Maryland, USA: Uganda National Malaria Control Division (NMCD), Uganda Bureau of Statistics (UBOS), and ICF.

Prieto-Patron, A., Horst, K. V., Hutton, Z. V., \& Detzel, P. (2018). Association between Anaemia in Children 6 to 23 Months Old and Child, Mother, Household and Feeding Indicators. Nutrients. 
UNICEF. (2021). Key practice: Malaria prevention. Retrieved September 6, 2021, from UNICEF: https://www.unicef.org/uganda/key-practice-malaria-prevention

WHO. (1968). Nutritional anaemias. Geneva: World Health Organization (WHO).

WHO. (2019). World Malaria Report 2019. Geneva: World Health Organisation.

\section{Figures}

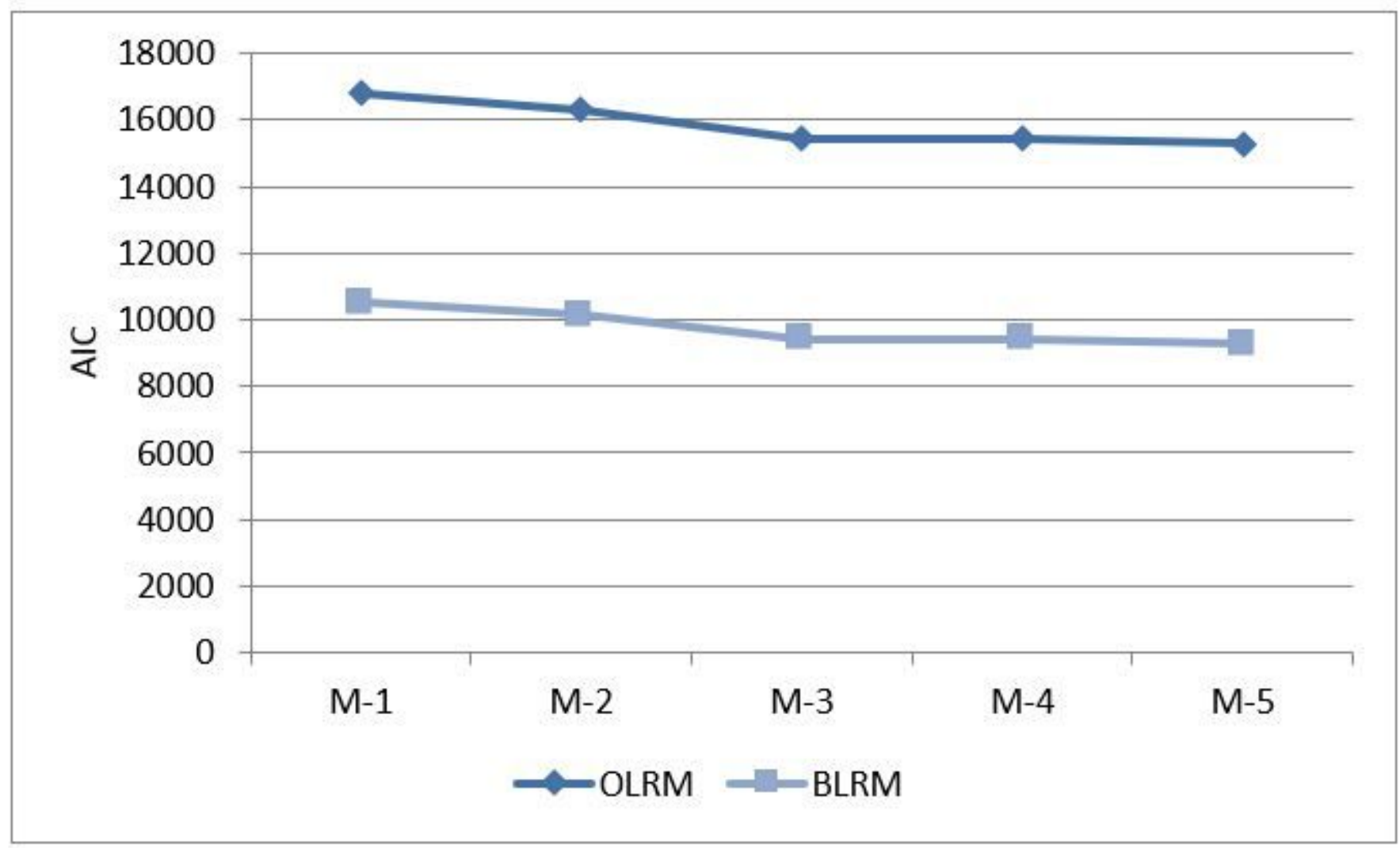

\section{Figure 1}

AIC values for estimates of ordered and binary (OLRM and BLRM) logistic regression models. 


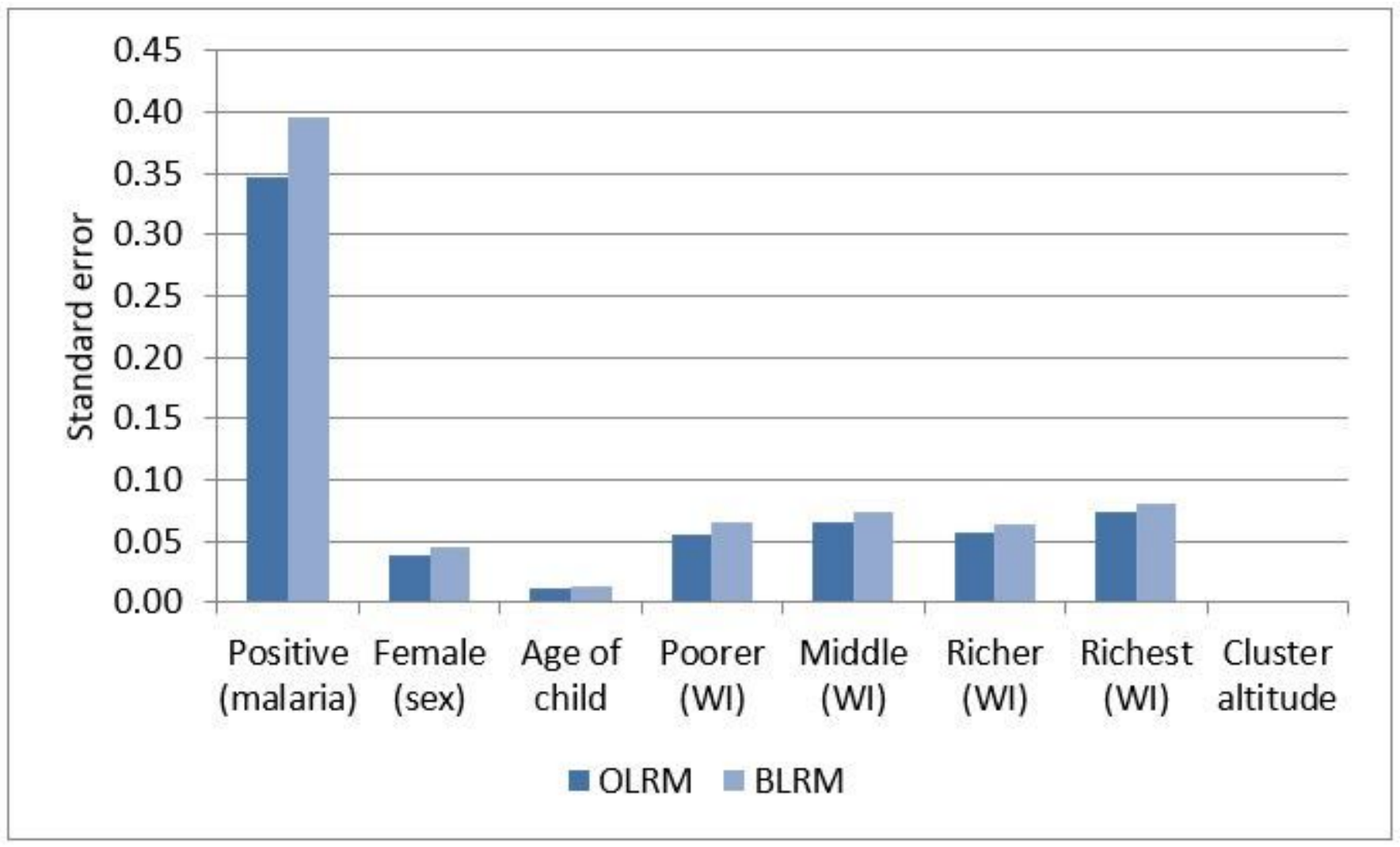

Figure 2

Standard errors of ordered and binary (OLRM and BLRM) logistic regression models. 